\title{
Flow Visualization of Aircraft in Flight by Means of Background Oriented Schlieren Using Celestial Objects
}

\author{
Michael A Hill ${ }^{1}$ and Edward A Haering Jr. ${ }^{2}$ \\ NASA Armstrong Flight Research Center, Edwards, California, 93523
}

\begin{abstract}
The Background Oriented Schlieren using Celestial Objects series of flights was undertaken in the spring of $\mathbf{2 0 1 6}$ at the National Aeronautics and Space Administration Armstrong Flight Research Center to further develop and improve a flow visualization technique which can be performed from the ground upon flying aircraft. Improved hardware and imaging techniques from previous schlieren tests were investigated. A United States Air Force T-38C and NASA B200 King Air aircraft were imaged eclipsing the sun at ranges varying from 2 to 6 nautical miles, at subsonic and supersonic speeds. Images were postprocessed using the Background Oriented Schlieren technique with optical flow algorithms to reveal both subsonic and supersonic flow features around the aircraft. The resulting imaging hardware and techniques demonstrated its applicability as a mature instrumentation system for performing flow visualization on vehicles in flight.
\end{abstract}

\section{Nomenclature}

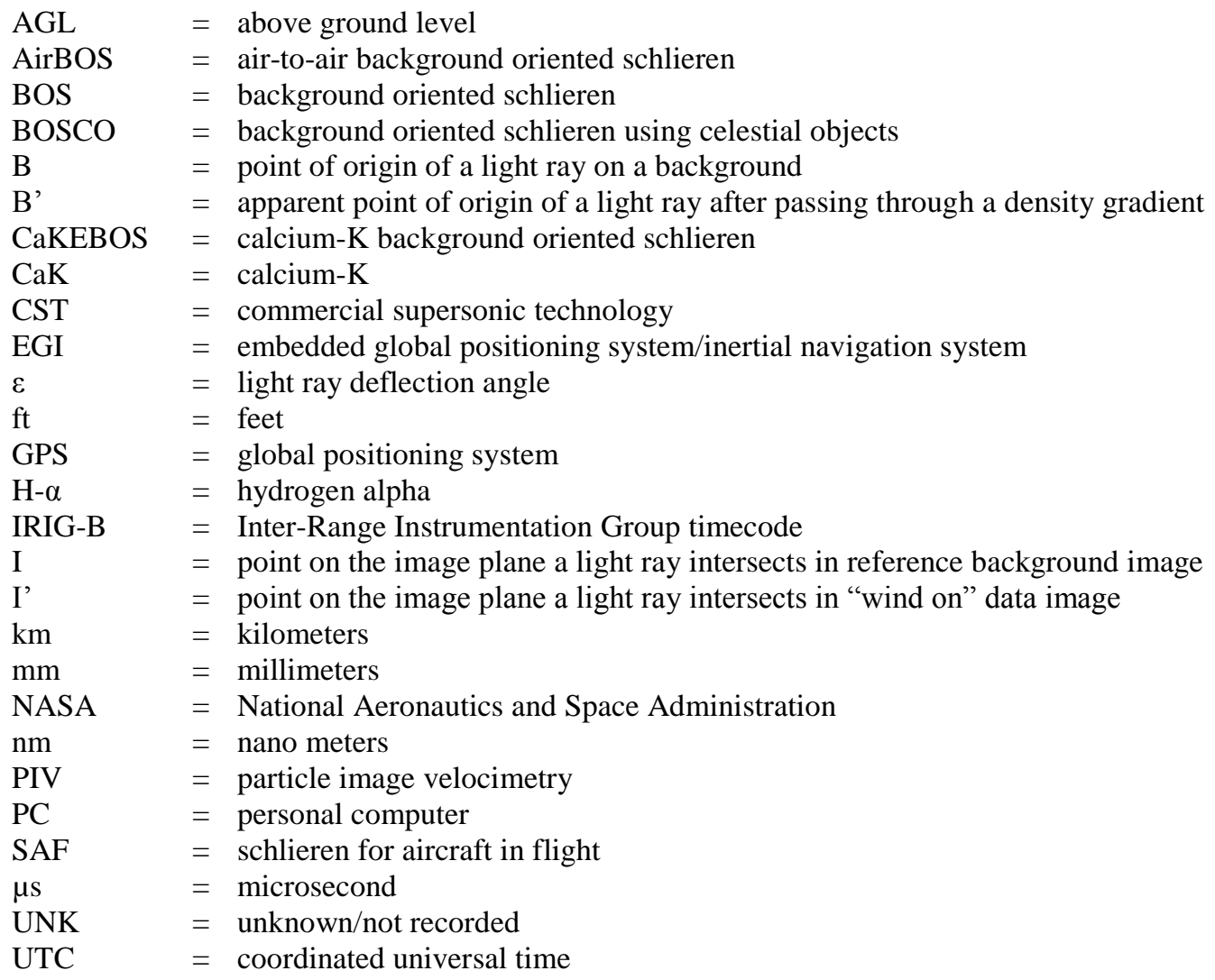

\footnotetext{
${ }^{1}$ Aerospace Engineer, Simulation, P.O. Box 273 / MS 4840A Edwards, California, AIAA nonmember.

${ }^{2}$ Aerospace Engineer, Research Aerodynamics and Propulsion Branch, P.O. Box 273 / MS 2228 Edwards, California, AIAA nonmember.
} 
WGS84 = World Geodetic System 1984

2D $\quad=$ two dimensional

$3 \mathrm{D}=$ three dimensional

\section{Introduction and Background}

$\mathrm{T}$ HE National Aeronautics and Space Administration (NASA) has been working for years with United States industry and academia to realize quiet boom civilian supersonic flight overland. The main technique used to quiet these aircraft involves shaping of the vehicle volume and lift distributions to prevent component shockwaves from combining to objectionable levels. While computational methods and wind tunnel studies are performed in the design of these vehicles, actual vehicle noise performance needs to be measured to prove success using both ground-level microphones and near-field measurements of shockwaves around the vehicle. In the event that a test vehicle produces a louder sound than designed, the near-field shock patterns can be used to diagnose the problem and offer direction for improvements. Flow visualization is an important tool in identifying these shock patterns.

Schlieren photography has been used for many years to visualize air density gradients caused by air compressing and expanding as it flows around an object. As the density of a gas changes, so does its refractive index, thus causing the light passing through it to bend. Traditionally, schlieren flow visualization uses complex and precisely aligned optics along with a background light source to cast shadows or create bright regions using these bent light rays. These diffracted rays appear in the schlieren image showing the intensity of the air density gradient around the object. Settles ${ }^{1}$ offers an extensive overview of schlieren techniques to date.

The Background Oriented Schlieren (BOS) method of flow visualization, was first demonstrated by Dalziel et al. ${ }^{2}$ and also Raffel et al. ${ }^{3}$ BOS has seen increased interest with the advancements in digital imaging and computational technology in recent years. BOS systems are relatively simple to setup when compared to traditional schlieren systems, and also offer a more complete picture of the light refraction in a single measurement.

The BOS technique, illustrated in Fig. 1, identifies ray deflection angles $(\varepsilon)$ by measuring displacement of features in a background image. An image of the reference background is taken first with minimal density gradients between the camera and the background. A light-refracting flow field is then introduced between the background image and the camera. The two images are compared and differences are attributed to refraction caused by density changes in the flow field. The measurement offers a view relating to the spatial gradient of fluid density, similar to the traditional schlieren system. The background that is imaged must have dense high contrast features which can be analyzed for pixel displacement. For wind tunnel and other ground based BOS experiments, the background is usually an image of randomly generated size-varying speckles that is placed behind the flow which is desired to be visualized. As shown in Fig. 1, with no density gradient, a light ray emanating from point B will hit at point I on the image plane. When passing through a change in air density, the ray from point B will bend (refract) and hit the image plane at point I'. This refraction will make it appear that point B is at point B'. By comparing digital images of the background through no density gradient with an image of the background through a gradient, distance $\Delta \mathrm{Y}$ can be found, and therefore, the refraction angle $\varepsilon$.

Pixel movement is commonly analyzed using cross-correlation algorithms which were developed for Particle Image Velocimetry (PIV) as described by Raffel et al. ${ }^{4}$ Optical flow algorithms developed for computer vision applications have also shown to be effective for BOS image processing. Atcheson et al., ${ }^{5}$ Smith et al., ${ }^{6}$ and Hill and Haering ${ }^{7}$ have all shown superior resolution of flow structures using these optical flow algorithms when compared to the PIV based cross correlation methods. 


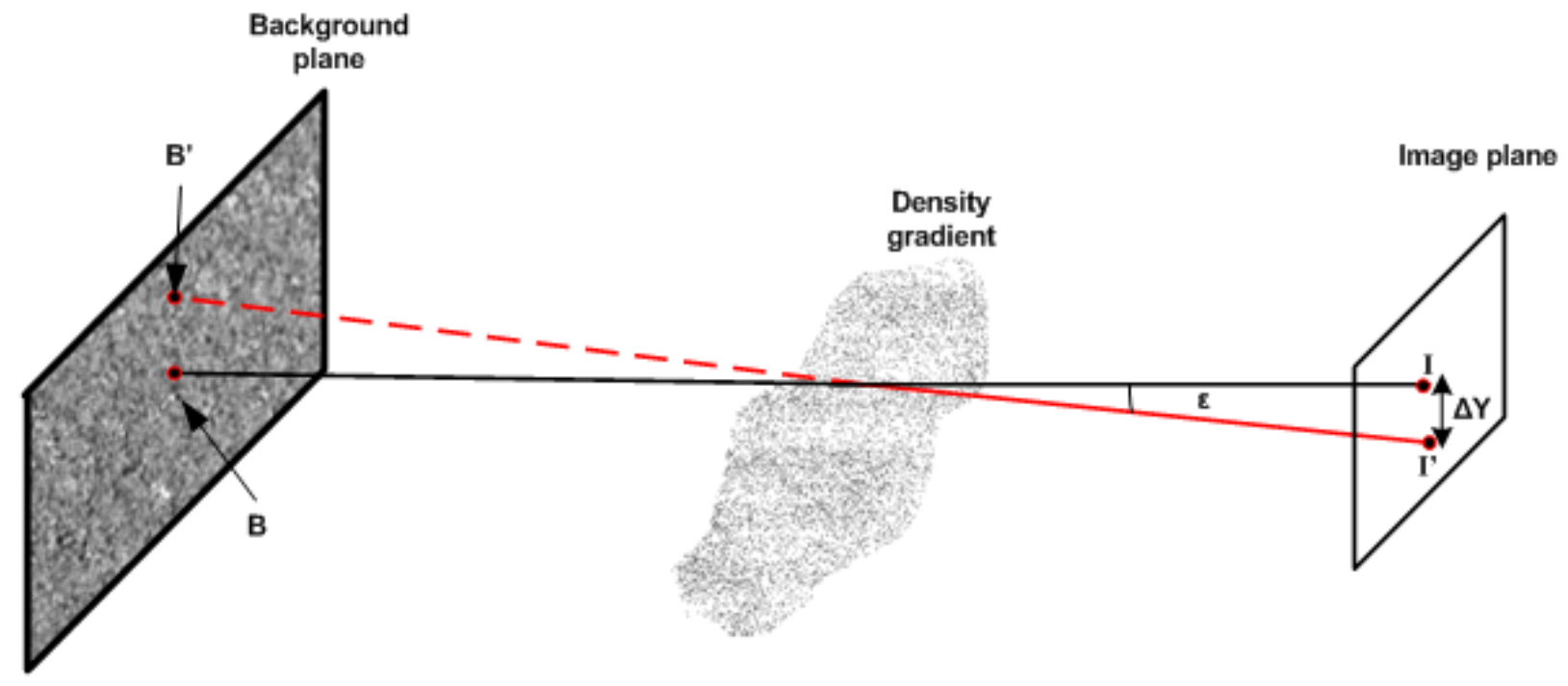

Figure 1. The BOS concept; by comparing digital images of the background through no density gradient with an image of the background through a gradient, distance $\Delta Y$ can be found and therefore the refraction angle $\varepsilon$.

\section{Schlieren on Flying Aircraft}

There have been a limited number of systems which have successfully performed schlieren on flying aircraft. Weinstein ${ }^{8}$ successfully demonstrated the Schlieren for Aircraft in Flight (SAF) system, which was a combination of a focusing schlieren system with a streak photography system, using the edge of the sun to obtain a streak image. Heineck et al..$^{9}$ demonstrated an air-to-air BOS system (AirBOS), in which an aircraft mounted camera, pointing downward, used the natural pattern of desert vegetation as a BOS background. The aircraft to be imaged flew beneath the camera aircraft, allowing for BOS to be performed on an aircraft in flight. Each of these schlieren systems has its advantages. The SAF system is much simpler and less costly to operate, being a ground based system. The AirBOS system has the advantages of being a BOS measurement, meaning that the data yields a full 2D map of pixel displacements, which allow measurement of refraction in any direction. The SAF system is limited to measuring density gradients perpendicular to the edge of the sun, which will hide any density gradients perpendicular to the direction of travel of the aircraft. AirBOS also offers the potential of higher resolution images due to the fact that the closer background uses much shorter focal length lenses, resulting in the ability to take images closer to the test aircraft. Certain advantages of both methods can be achieved by combining the BOS measurement with the ground based SAF system.

In April 2015 the Calcium-K Eclipse Background Oriented Schlieren (CaKEBOS) series of tests was undertaken to test the applicability of using the optically filtered image of the sun as a background for BOS. ${ }^{7}$ CaKEBOS utilized a calcium-K (CaK) optical band pass filter to view the granulation cells on the chromosphere of the sun. Aircraft eclipsed the sun relative to a ground based imaging system. Density gradients caused by the flow around the aircraft causing the light to refract and the image of the granulation cells on the chromosphere of the sun to change position on the camera sensor. The movement of the texture of the solar background allowed for processing the images using the BOS method. CaKEBOS proved the concept of Background Oriented Schlieren using Celestial Objects (BOSCO), which is illustrated in Fig. 2 in a single dimension for simplicity. A ray from the sun passing through an undisturbed atmosphere will intersect the image plane at point I. If that ray passes through a density gradient, it will refract at angle $\varepsilon$ and intersect the image plane at point I'. Obtaining images of the solar chromosphere without the density gradient yields the location of point I, with images taken though the density gradient giving the location of point I'. Optical flow algorithms calculate the displacement in each direction $\Delta \mathrm{Y}$ and $\Delta \mathrm{X}$. Mapping the magnitudes of these displacements to a grayscale image yields the final schlieren image. 


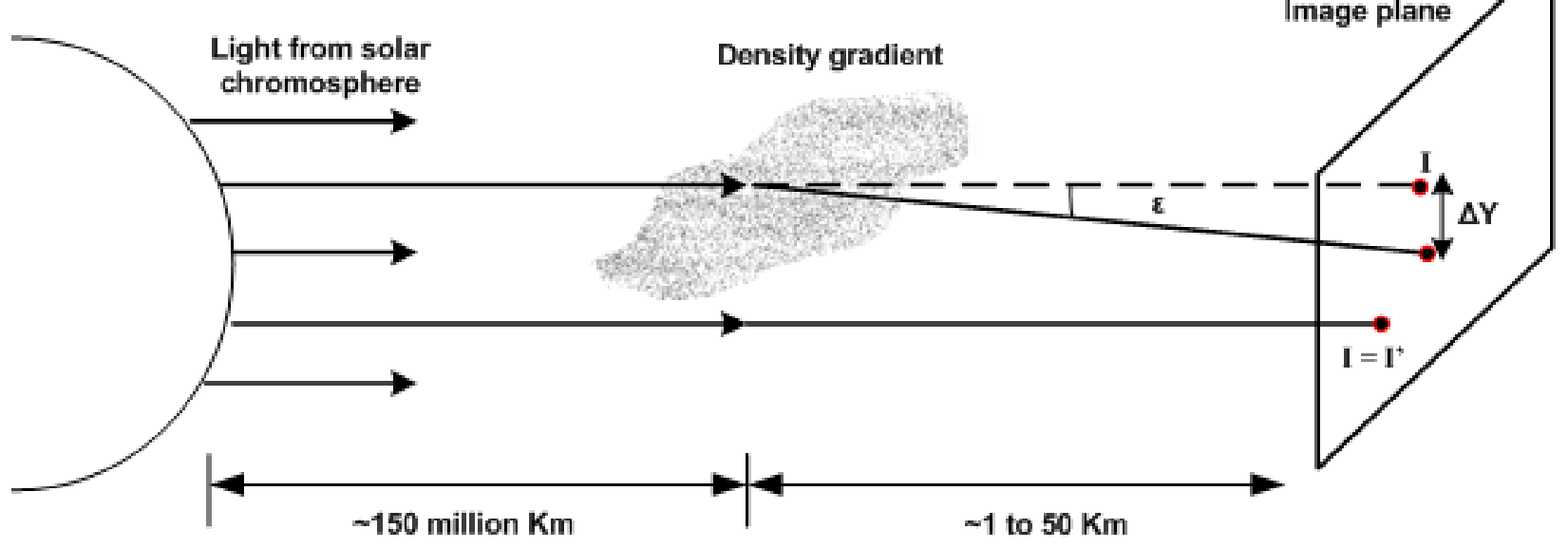

Figure 2. The BOSCO concept: an atmospheric density gradient will cause light from the sun to refract, causing a light ray that would normally intersect the image plane at point I, to intersect the plane at point I'.

\section{BOSCO Experimental Setup}

The BOSCO flights undertaken in spring of 2016 were intended to incrementally improve the ground-to-air BOS technology which CaKEBOS demonstrated was possible. With the 2016 flights, the camera and optical equipment was upgraded from the imager used for CaKEBOS, and a different solar optical filter, the hydrogen- $\alpha(\mathrm{H}-\alpha)$ emission line was investigated. Three flights took place, one flight imaging a NASA Beechcraft King Air aircraft (Beechcraft Corporation, Wichita, Kansas), and two flights to image a U.S. Air Force T-38C aircraft (Northrop Corporation, Hawthorne, California) at both supersonic and subsonic speeds.

The basic concept of a BOSCO measurement is shown in Fig. 3, the new system being identical to the system used for CaKEBOS, with the exception of the upgraded ground hardware and change in optical filter. It has been shown by Atcheson et al. ${ }^{5}$ that backgrounds with a wide range of spatial frequencies work well for BOS. These different spatial frequencies allow for a wide range camera to target distances without reducing the sensitivity of the system. Several consumer solar optical filters exist, with the most popular being the 394-nm wavelength CaK filter used for CaKEBOS, and the $\mathrm{H}-\alpha$ emission line which passes the $656.28-\mathrm{nm}$ wavelength of hydrogen on the solar chromosphere. Images of the sun through the two filters are compared in Fig. 4. Basic analysis yields that images of the H- $\alpha$ emission line of the sun have a wider range of spatial frequencies than images of the CaK line which suggested that the $\mathrm{H}-\alpha$ images may be better suited to a wider range of BOS analysis. It is also apparent in Fig. 4 that the features in the H- $\alpha$ image are much denser and have a higher contrast when compared to the CaK image. 


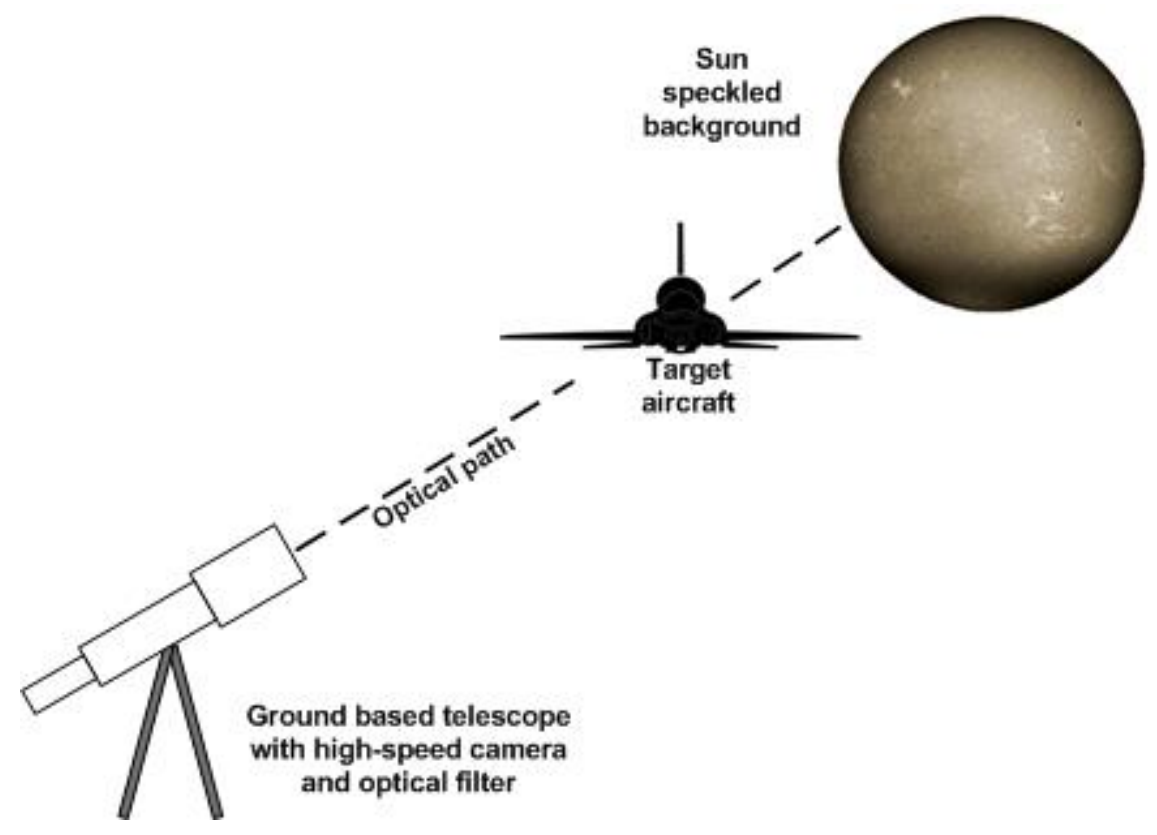

Figure 3. BOSCO test; an aircraft eclipses the sun relative to the imager. The flow field around the aircraft causes density gradients that refract the light, causing apparent movement of texture on the sun.

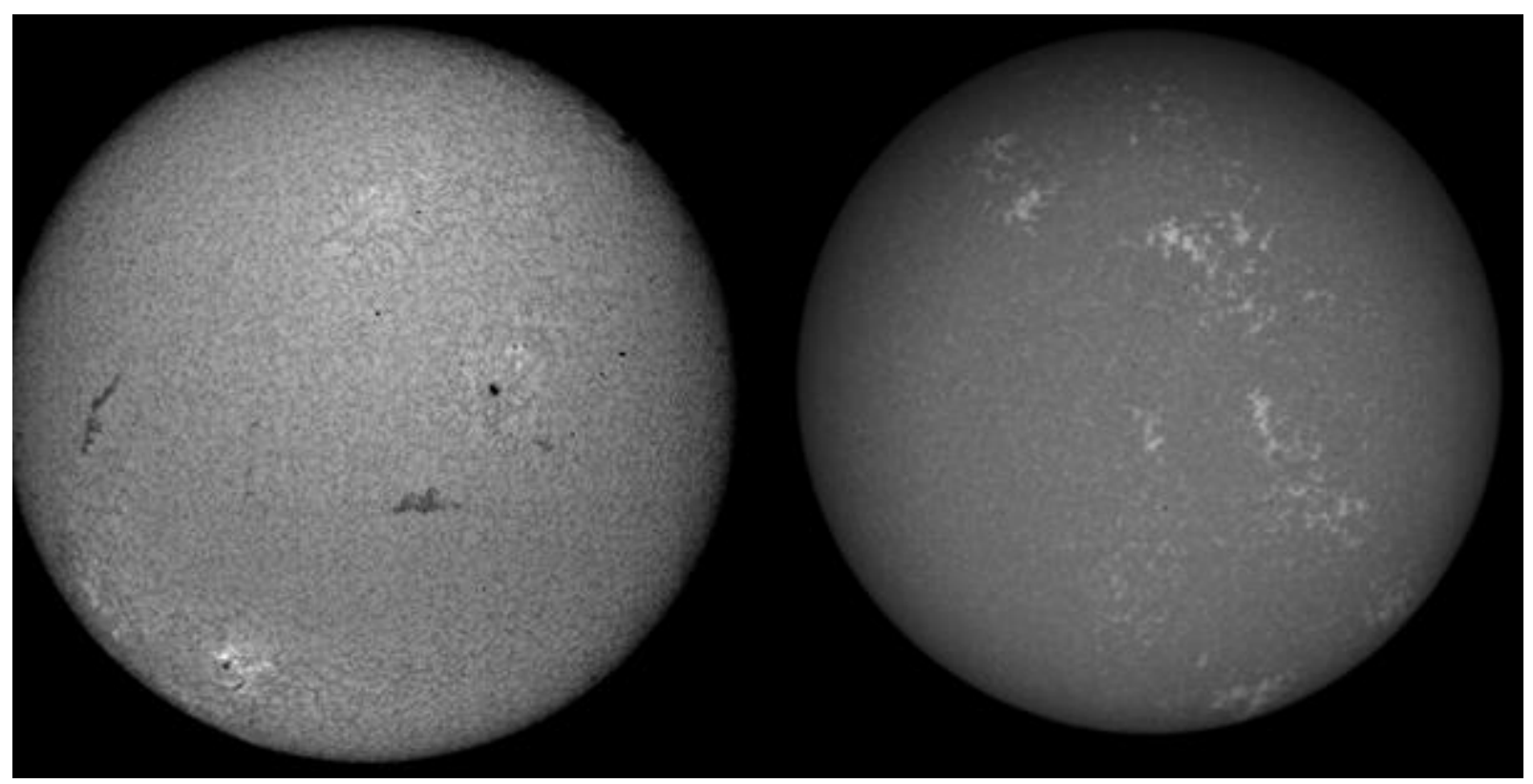

Figure 4. The H- $\alpha$ line (left); CaK line (right).

The imaging system, Fig. 5, consists of the primary lens with integrated H- $\alpha$ filter, Lunt LS100THA (Lunt solar systems, Tuscon, Arizona), that is coupled with a $3 \mathrm{X}$ focal extender (Explore Scientific, Springdale, Arkansas) for an effective focal length of roughly $2100 \mathrm{~mm}$. This focal length, when coupled with the Photron WX100 high-speed camera (Photron Inc., Tokyo, Japan), makes maximum use of the camera sensor with the solar disk image 1,970 pixels in diameter for a 2,048 X 2,048 pixel sensor. A Celestron CGEM equatorial mount (Celestron Inc., Torrance, California) performs open-loop tracking of the sun. The camera is controlled with a Windows (Microsoft Corporation, Redmond, Washington) laptop and is fed an Inter-range Instrumentation Group (IRIG-B) time code in order to 
accurately timestamp the images for post-flight analysis. The camera records in a circular buffer, which saves roughly 2 seconds before and after the trigger for a total of 4 seconds of 12-bit images at 1000 frames per second.

The focal length of the lens yields 0.5 -degree field of view, which translates to roughly $300-\mathrm{ft}$ field of view at $30,000 \mathrm{ft}$ AGL. To minimize aircraft blurring, the integration time (shutter speed) of the camera was minimized while providing an ample signal. The speed of the aircraft coupled with the camera field of view allowed for the calculation of the number of pixels the aircraft would pass over while the shutter was open. An integration time of $333 \mu$ s resulted in the aircraft travelling over a maximum of 2 pixels during the sensor integration time for a pass at 30,000 $\mathrm{ft}$ AGL at Mach 1.05. The maximum pixel brightness value of the solar image for this integration time was 1800 out of 4095 for the 12-bit sensor, with an average value of 1400 . This signal level coupled with the minimal aircraft movement while the shutter was open, was seen as a good compromise to minimize the blurring of the aircraft during the eclipse.

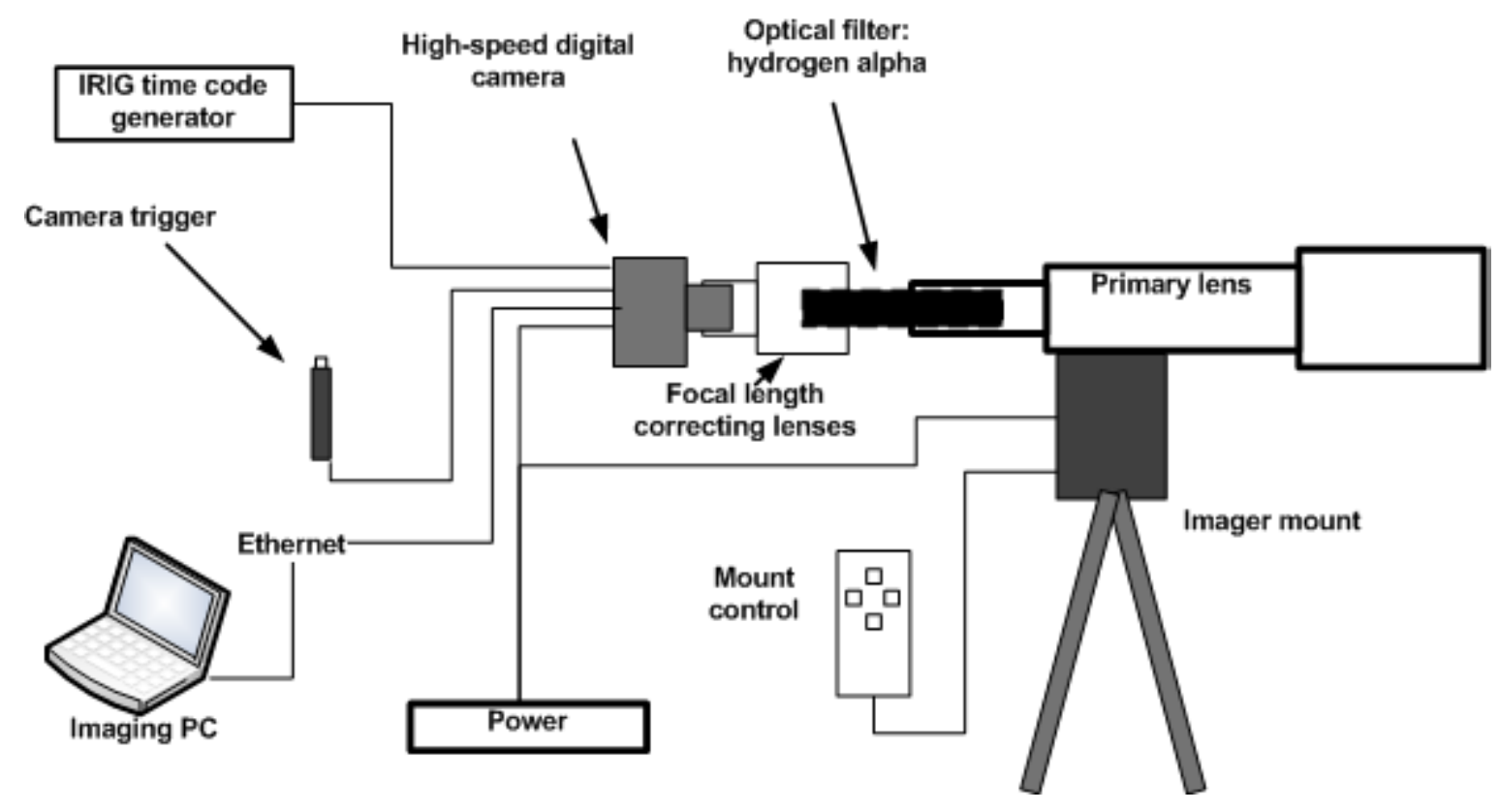

Figure 5. BOSCO ground imaging system block diagram and photo.

To increase the vertical field of view of the imaging system, three imagers were used in an array as shown in Fig. 6. Because of hardware availability limitations, the B and C telescopes used a lower resolution Hispec 2 camera (Fastec Imaging, San Diego, California) and a CaK filter (Lunt Solar, Tuscon, Arizona). These imagers are described in more detail by Hill and Haering. ${ }^{7}$ Due to the extreme distance from the flow feature to the background, it was assumed the look angle to the sun of all of the imagers are identical, even though they are spaced apart on the ground. At 30,000 ft altitudes (AGL), the 1/2-degree field of view of each imager with 10-\% overlap at the target altitude will yield a total field of view of roughly $850 \mathrm{ft}$.

The imagers were located on the NASA Armstrong Flight Research Center (Edwards, California) campus to make use of the high altitude supersonic corridor located in the Edwards Air Force base R-2508 airspace complex. The three imagers are shown in Fig. 7 as (A), which was the primary imager, and (B) which was the north imager, and (C) which was the south imager. 


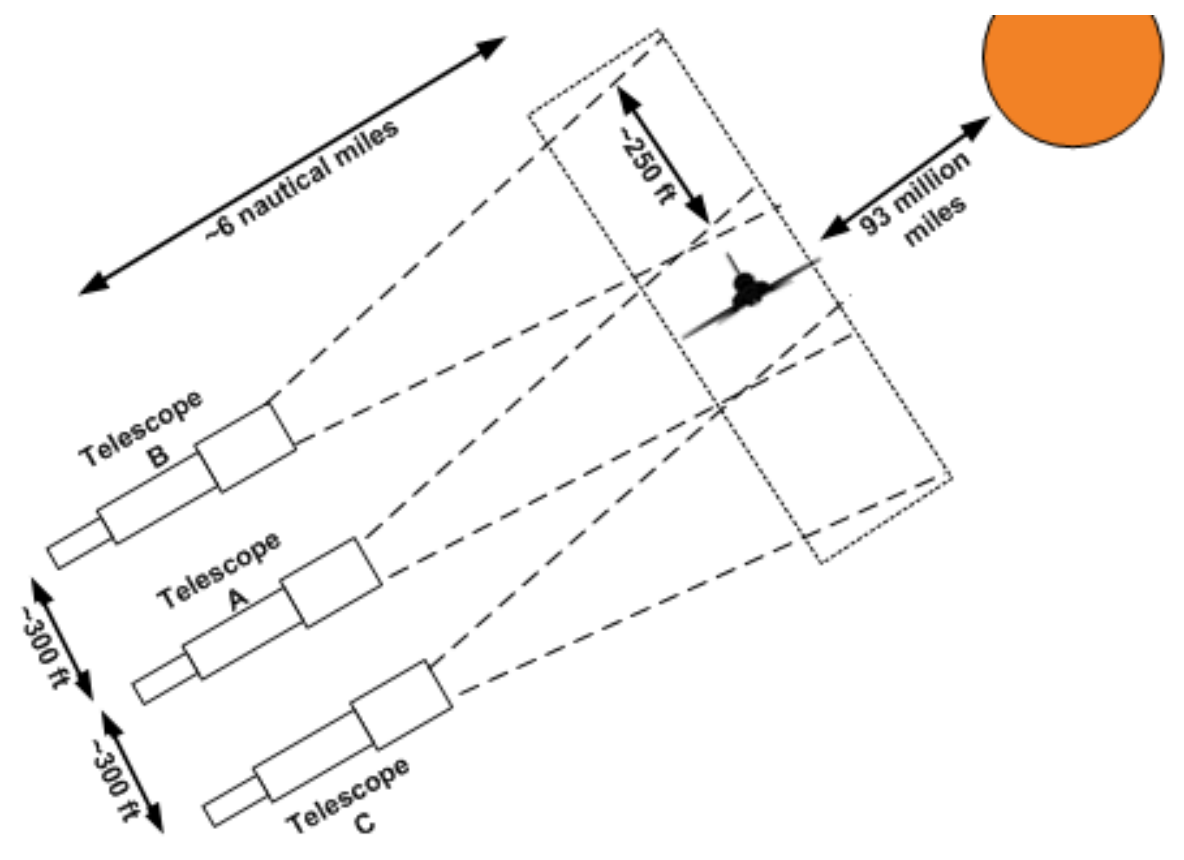

Figure 6. Three imager array concept for increasing field of view.

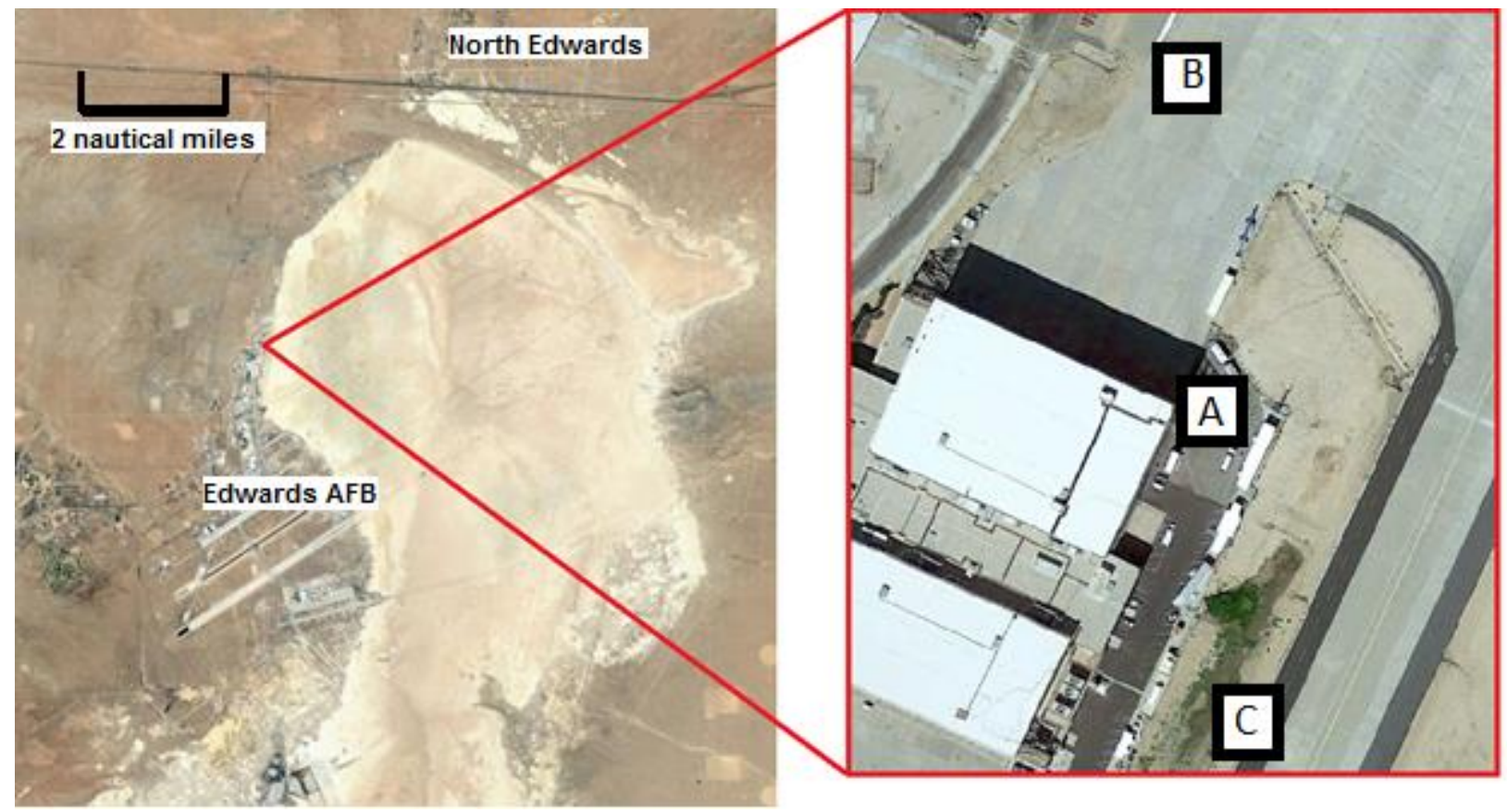

Figure 7. Location for the ground equipment for the spring 2016 BOSCO test. Markers (A), (B), and (C) indicate the primary, north, and south imaging systems respectively.

\section{Flight-Test Methods}

Two flights with a total of 11 passes have been performed with a U.S. Air Force T-38C aircraft, and one flight with one pass was completed with a Beech B200 King Air aircraft. The two T-38 aircraft flights included both supersonic and subsonic runs. Nominal supersonic flight conditions had the T-38 aircraft fly at 32,000 ft ellipsoid 
global positioning system (GPS) altitude at Mach 1.05. Subsonic passes for both the T-38 and the King Air aircraft were flown at 12,500 ft GPS altitude. A summary of the passes and results are shown in Tables 1a to 1c.

Successful collection of BOSCO data depended on accurate waypoint planning to ensure the aircraft would eclipse the sun relative to the imager. Aircraft waypoints, shown in Fig. 8, were preplanned based on the location of the imager on the ground and the expected eclipse time. A solar position calculation algorithm ${ }^{10}$ was used to find the elevation and azimuth of the sun for a given time. Aircraft waypoints were found by selecting an altitude for flight, then using standard trigonometric formulas ${ }^{11}$ to determine the World Geodetic System 1984 (WGS84) latitude and longitude for the waypoint the aircraft as described by Hill and Haering. ${ }^{7}$ Waypoints were calculated every 2 minutes, but are shown in Fig. 8 at ten-minute spacing for simplicity. To minimize the timing sensitivity of hitting the waypoint, the course of the aircraft followed the course of the current waypoint to the next waypoint, as shown in Fig. 8 for the March 24 waypoints. Flying this course enabled the aircraft to eclipse the sun relative to the imager as long as it hit the waypoint within \pm 2 minutes of the expected time.

Navigation equipment in the T-38 aircraft consisted of the Honeywell H-764G (Honeywell International, Morris Plains, New Jersey) embedded global positioning system/inertial navigation system (EGI). A Garmin 596 (Garmin corporation, Schaffhausen, Switzerland) GPS system was used in the King Air aircraft to navigate to the waypoints. Cameras were manually triggered on radio call by the pilots or by visual eclipse as seen by the imager operator. Shown in Fig. 8, as the sun elevation increases with the changing dates, and the aircraft altitude lowers, the waypoint becomes closer to the camera. As aircraft altitude increases and when the sun elevation is low, the points are farther from the camera.

Table 1. Summary of the 2016 BOSCO flight tests.

Table 1a. Flight 1: King Air aircraft 3/10/2016.

\begin{tabular}{|c|c|c|c|c|c|c|}
\hline \hline Pass & $\begin{array}{c}\text { Time } \\
(\mathrm{UTC})\end{array}$ & $\begin{array}{c}\text { Camera range } \\
\text { (Nautical miles) }\end{array}$ & $\begin{array}{c}\text { Sun } \\
\text { elevation / } \\
\text { Azimuth }\left({ }^{\circ}\right)\end{array}$ & $\begin{array}{c}\text { Altitude (ft GPS) / } \\
\text { Mach / } \\
\text { True course }\end{array}$ & Test point & $\begin{array}{c}\text { Imager } \\
\text { eclipsed }\end{array}$ \\
\hline 1 & $20: 30$ & 2.21 & $51 / 192$ & $12,500 /$ UNK / UNK & $\begin{array}{c}\text { Flaps } \\
\text { down }\end{array}$ & $\mathrm{A}$ \\
\hline \hline
\end{tabular}

Table 1b. Flight 2: T-38C aircraft 3/24/2016.

\begin{tabular}{|c|c|c|c|c|c|c|}
\hline \hline Pass & $\begin{array}{c}\text { Time } \\
\text { (UTC) }\end{array}$ & $\begin{array}{c}\text { Camera range } \\
\text { (Nautical miles) }\end{array}$ & $\begin{array}{c}\text { Sun } \\
\text { elevation } / \\
\text { Azimuth }\left({ }^{\circ}\right)\end{array}$ & $\begin{array}{c}\text { Altitude (ft GPS) / } \\
\text { Mach / } \\
\text { True course }\end{array}$ & Test point & $\begin{array}{c}\text { Imager } \\
\text { eclipsed }\end{array}$ \\
\hline 1 & $19: 15: 21$ & 5.94 & $55 / 161$ & $\begin{array}{c}32,095 / 1.05 \mathrm{M} / \\
269\end{array}$ & $\begin{array}{c}\text { Straight and } \\
\text { level }\end{array}$ & $\mathrm{A}$ \\
\hline 2 & $19: 23: 11$ & 5.94 & $56 / 164$ & $\begin{array}{c}32,008 / 1.05 \mathrm{M} / \\
269\end{array}$ & $\begin{array}{c}60^{\circ} \text { right } \\
\text { bank }\end{array}$ & $\mathrm{C}$ \\
\hline 3 & $19: 30: 15$ & 5.94 & $56 / 168$ & $\begin{array}{c}32,074 / 1.06 \mathrm{M} / \\
269\end{array}$ & $\begin{array}{c}60^{\circ} \text { right } \\
\text { bank }\end{array}$ & $\mathrm{C}$ \\
\hline 4 & $19: 37: 15$ & 5.94 & $57 / 171$ & $\begin{array}{c}32,074 / 1.05 \mathrm{M} / \\
270\end{array}$ & $\begin{array}{c}60^{\circ} \text { right } \\
\text { bank }\end{array}$ & $\mathrm{B}$ \\
\hline 5 & $19: 46: 35$ & 2.0 & $57 / 175$ & $\begin{array}{c}12,535 / 0.26 \mathrm{M} / \\
270\end{array}$ & $\begin{array}{c}\text { Level flaps } \\
\text { down }\end{array}$ & $\mathrm{A}$ \\
\hline 6 & $19: 52: 15$ & 2.0 & $57 / 179$ & $\begin{array}{c}12,512 / 0.26 \mathrm{M} / \\
270\end{array}$ & $\begin{array}{c}\text { Level flaps } \\
\text { down }\end{array}$ & None \\
\hline \hline
\end{tabular}


Table 1. Concluded.

Table 1c. Flight 3: T-38C aircraft 4/5/2016.

\begin{tabular}{|c|c|c|c|c|c|c|}
\hline \hline Pass & Time (UTC) & $\begin{array}{c}\text { Camera range } \\
\text { (Nautical miles) }\end{array}$ & $\begin{array}{c}\text { Sun } \\
\text { elevation / } \\
\text { Azimuth }\left({ }^{\circ}\right)\end{array}$ & $\begin{array}{c}\text { Altitude (ft GPS) / } \\
\text { Mach / } \\
\text { True course }\end{array}$ & Test point & $\begin{array}{c}\text { Imager } \\
\text { eclipsed }\end{array}$ \\
\hline 1 & $19: 13: 57$ & 5.73 & $60 / 159$ & $\begin{array}{c}31,945 / 1.04 \mathrm{M} / \\
269\end{array}$ & $\begin{array}{c}\text { Straight and } \\
\text { level }\end{array}$ & $\mathrm{A}$ \\
\hline 2 & $19: 27: 38$ & 5.62 & $61 / 167$ & $\begin{array}{c}31,908 / 1.04 \mathrm{M} / \\
269\end{array}$ & $\begin{array}{c}\text { Straight and } \\
\text { level }\end{array}$ & $\mathrm{C}$ \\
\hline 3 & $19: 35: 24$ & 5.60 & $61 / 171$ & $\begin{array}{c}32,002 / 1.06 \mathrm{M} / \\
269\end{array}$ & $\begin{array}{c}60^{\circ} \text { right } \\
\text { bank }\end{array}$ & $\mathrm{A}$ \\
\hline 4 & $19: 42: 27$ & 5.59 & $61 / 174$ & $\begin{array}{c}31,920 / 1.05 \mathrm{M} / \\
269\end{array}$ & $\begin{array}{c}60^{\circ} \text { right } \\
\text { bank }\end{array}$ & $\mathrm{A}$ \\
\hline 5 & $19: 49: 59$ & 1.93 & $61 / 178$ & $\begin{array}{c}12,500 / 0.26 \mathrm{M} / \\
270\end{array}$ & $\begin{array}{c}\text { Level } \\
\text { flaps down }\end{array}$ & $\mathrm{A}$ \\
\hline \hline
\end{tabular}

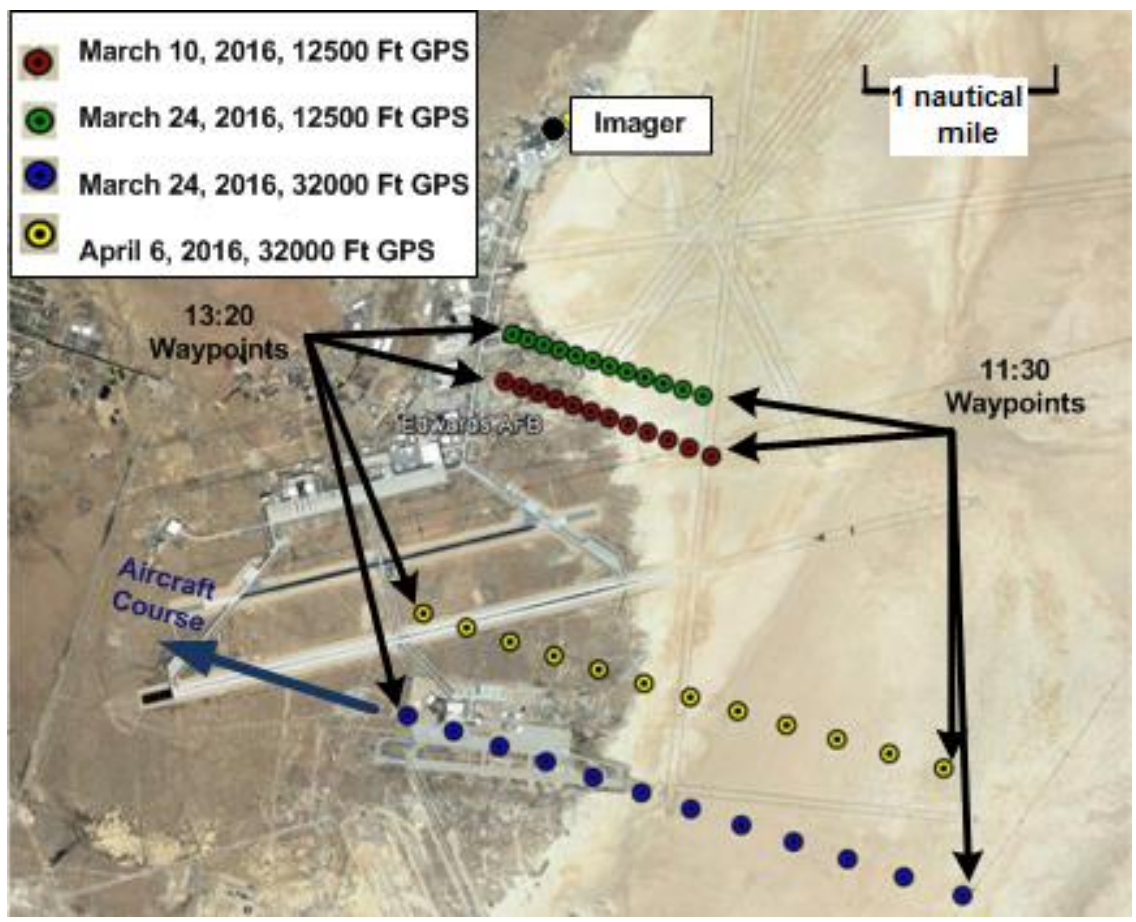

Figure 8. Aircraft waypoints for BOSCO flights. Waypoints to eclipse the sun relative to the imager changed with date, time, and aircraft altitude.

\section{Data Analysis and Results}

Representative unprocessed eclipse images are shown for flight 1 passes 1 and 5 in Fig. 9. The T-38 aircraft at $12,500 \mathrm{ft}$ GPS altitude appears much larger on the solar disk than the T-38 aircraft at the much longer range at 32,000 ft GPS altitude. In the right image the T-38 aircraft partially eclipsed the solar disk to the right. 


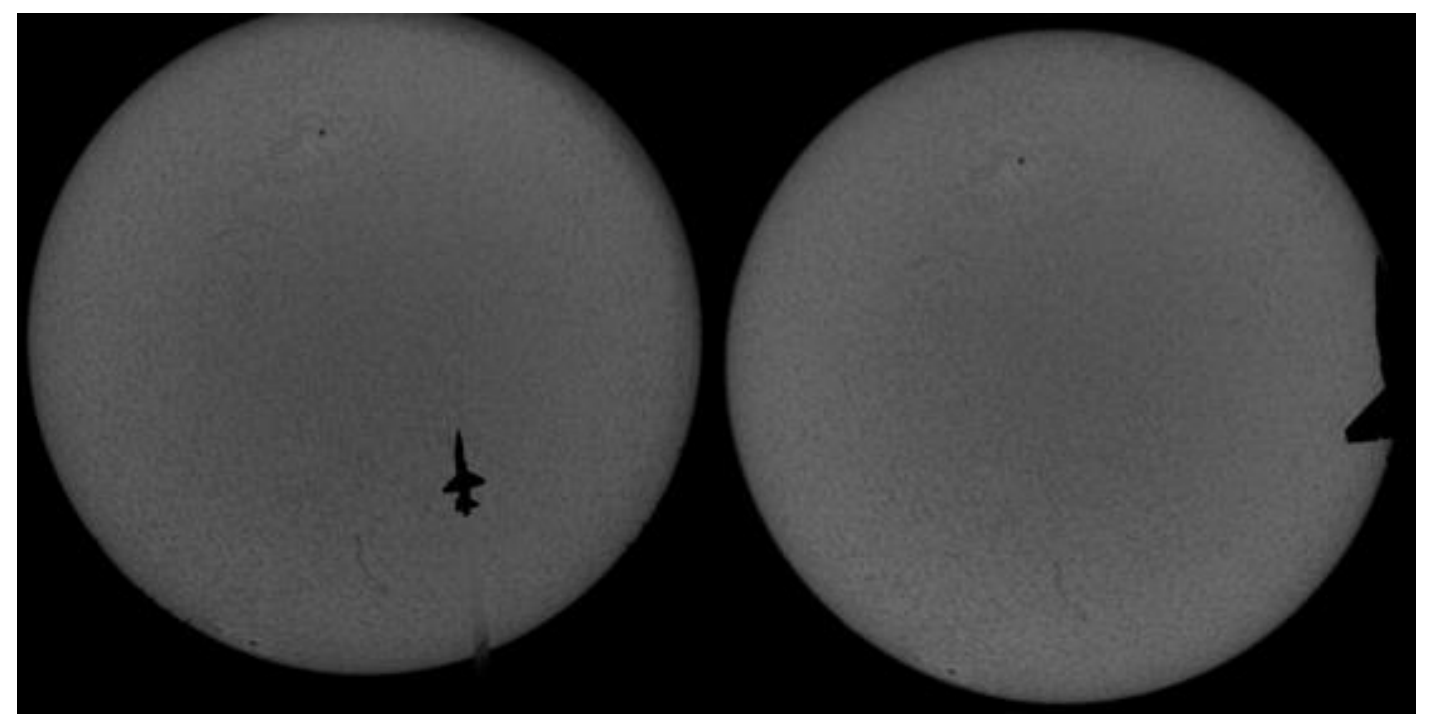

Figure 9. Left: T-38 aircraft at 32,000 ft GPS altitude. Right: T-38 aircraft eclipsing the edge of the solar disk at $12,500 \mathrm{ft}$ GPS altitude.

Raw images collected of the eclipse were processed using optical flow algorithms provided in the OpenCV (openCV.org) package for Python (python.org). In order to reduce noise caused by natural atmospheric turbulence, the 10 image frames before the aircraft entered the field of view were used for the reference images of the background in which no density gradient is present. Each image with the aircraft eclipsing was aligned to the reference background to account for camera shake. Lucas-Kanade ${ }^{12}$ optical flow as implemented in OpenCV 2.0 was then performed on each image with the aircraft eclipsing against the 10 backgrounds. The 10 results for pixel deflections were then averaged by taking the median deflection at each pixel for the 10 solutions. By taking the median of the 10 solutions from the 10 backgrounds, one solution was found for each frame with the aircraft eclipsing. For further noise reduction, the median pixel deflection for 100 frames of the aircraft eclipsing were then taken, giving the final schlieren image. Since BOS yields 2D vectors of pixel deflection, schlieren images at any "knife edge" orientation can be taken from a single dataset. The knife edge angle, which is named for the knife edge used in traditional schlieren systems, is the angle on the image plane perpendicular to which the gradients in density are seen. For example, a knife edge along the longitudinal axis of the aircraft will show density gradients in the lateral direction. The schlieren images shown in Fig. 10 are 2D representations of a 3D flow field and represent the path-integrated change in density in the direction perpendicular to the knife edge.

Figure 10 shows a comparison of the processed schlieren images of supersonic passes using different solar filters with camera and other optics effectively unchanged. The knife-edge orientation is perpendicular to the direction of the aircraft.

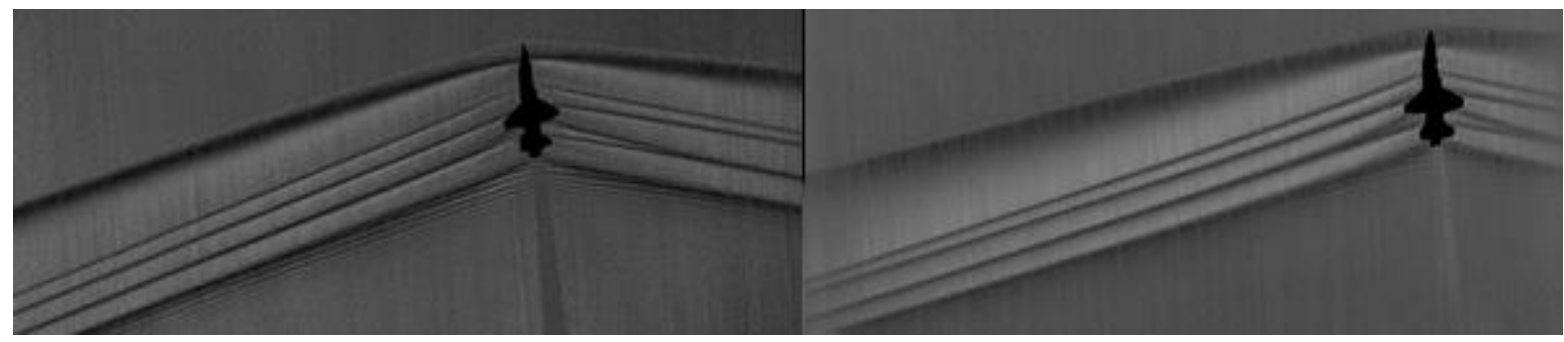

Figure 10. Left: H-a flight 2, pass 1. Right: CaK flight 3, pass 2.

It can be seen qualitatively from Fig. 10 that as expected, the $\mathrm{H}-\alpha$ filter provides a better background for BOS analysis, yielding lower noise and higher detail schlieren images when compared to the CaK filter. With the new system, the resolution is no longer driven by the digital pixel resolution as the smallest visable shock structures that are visable cover multiple pixels. 
The concept of using a 3 camera array as a method to obtain a large field of view was found to be effective as shown in Fig. 11, which is flight 1 pass 4, with side views being obtained by banking the aircraft at the solar elevation angle. In Fig. 11, the higher resolution $\mathrm{H}-\alpha$ system is located in the center, with the CaK telescopes in the top and bottom. With the combination of the three imager array and aircraft banking, shock structure can be seen 11 body lengths below the aircraft.

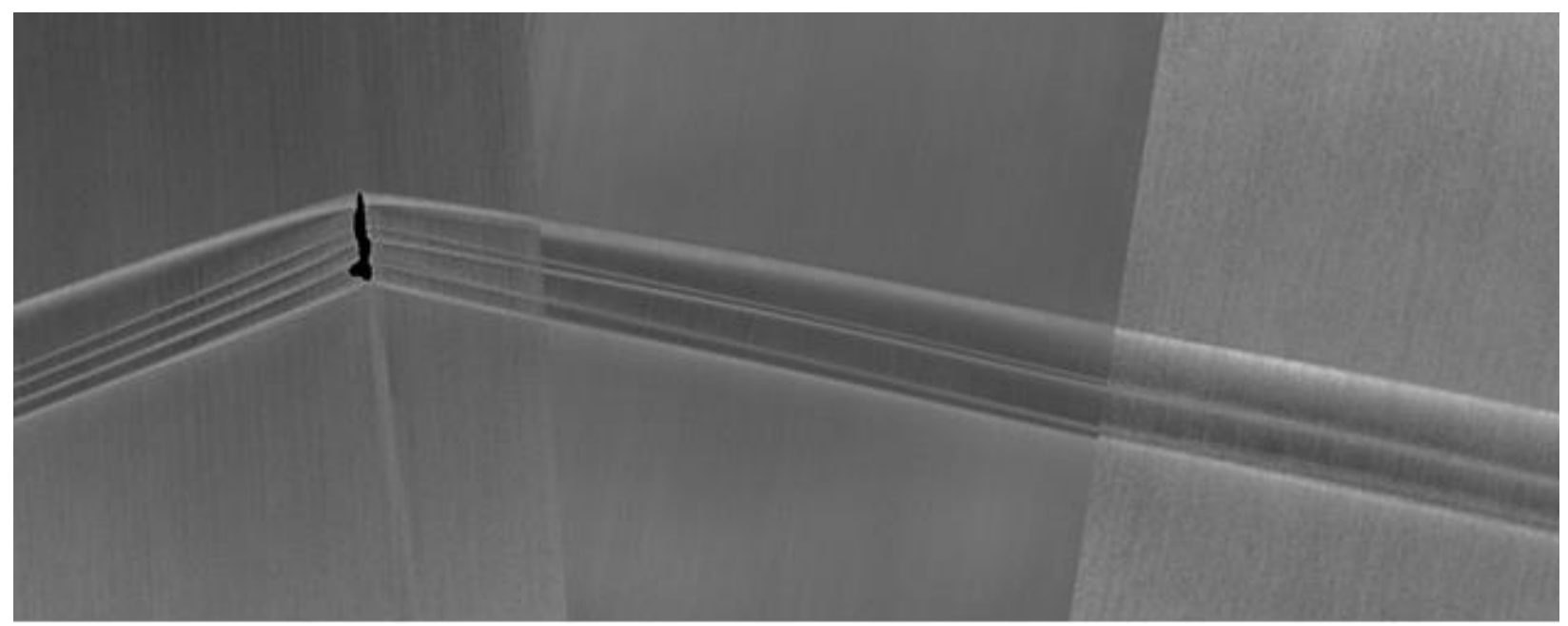

Figure 11. Three imager mosaic of flight 1, pass 4; imager B (left), imager A (center), and imager C (right).

Subsonic flow features were also imaged. Wing tip and flap vortices from a subsonic T-38 aircraft for flight 2, pass 5 is shown in Fig. 12. The aircraft did not hit the waypoint exactly on the pass, which resulted in a partial eclipse. Because of the partial eclipse by the T-38 aircraft, only $1 / 2$ of the aircraft is visible in the schlieren image. At the roughly 2-mile range of Fig. 12, the wingtip and flap vortex is clearly visible.

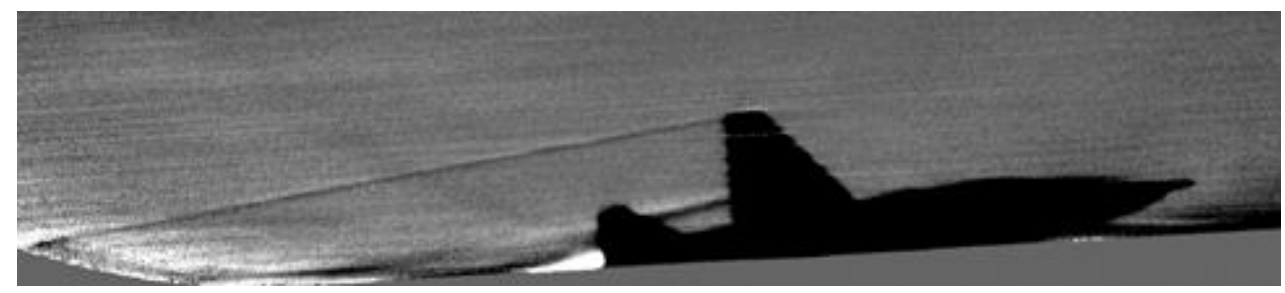

Figure 12. Wing tip and flap vortex from a subsonic T-38 aircraft; 12,500 ft GPS altitude; flight 2, pass 5.

Figure 13 shows the processed pass performed by the King Air aircraft at approximately the 2-mile range. Unfortunately the camera sensor was not properly black-level calibrated prior to the pass shown in Fig. 13, which resulted in very high noise raw images. Even with very poor quality raw data, a flap vortex and a faint wingtip vortex is visible. 


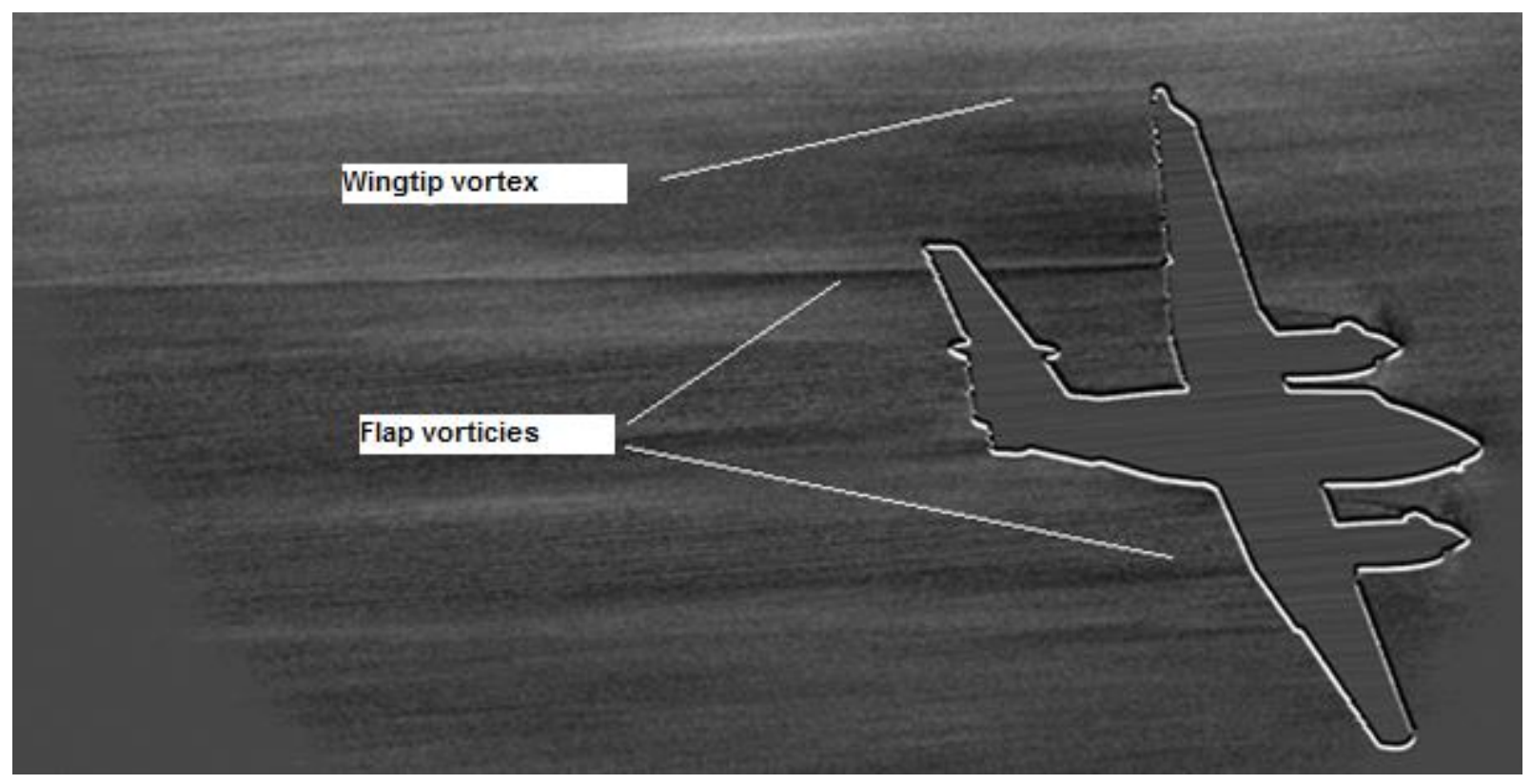

Figure 13. Processed BOS image of a Beechcraft King Air aircraft at approximately the 2-mile range; flight 1, pass 1. A calibration problem with the high-speed camera caused a high noise in the raw data images.

Finally, Fig. 14 shows the same pass as shown in Fig. 10, flight 2 pass 1, with the schlieren knife edge rotated to be parallel with the aircraft longitudinal axis. Rotating the knife edge allows for the viewing of density gradients in the direction perpendicular to the aircraft such as the wingtip vortex. By processing many extra frames of after the aircraft had eclipsed, an extended view behind the aircraft can be seen. Unfortunately, the short wings on the T-38 aircraft cause the vortex to quickly be overwhelmed by the engine exhaust, greater than 5 body lengths aft.

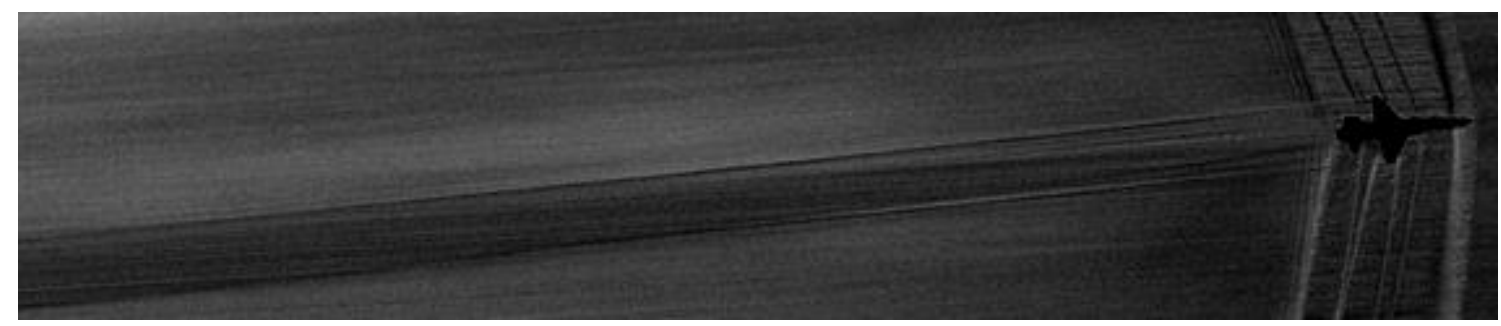

Figure 14. T-38 aircraft at 32,000 ft GPS altitude at Mach 1.05; longitudinal schlieren knife edge; flight 2, pass 1.

\section{Discussion and Future work}

The latest set of Background Oriented Schlieren using Celestial Objects flights improved on the technology initially demonstrated in the Calcium-K Background Oriented Schlieren flights. It was shown that a higher resolution camera (2048X2048 pixel) will improve the detail of the final schlieren image. As previously stated, the smallest shock structures that were seen in the schlieren images are multiple pixels in thickness, which suggests that the system is now limited by its optical sensitivity at the current ranges of 3 miles and longer, and that a higher digital resolution camera than the current one will not yield any more detail in the final schlieren image.

It was also demonstrated that the solar disk as viewed through the hydrogen- $\alpha$ filter provides a better suited Background Oriented Schlieren background than the calcium-K filter. The multiple imager array was shown to be an effective technique to increase the field of view, and that processing frames after the aircraft eclipse can yield an extended view of the flow field behind the aircraft.

The ground based Background Oriented Schlieren using Celestial Objects system is now a mature and deployable instrumentation system that can be used to perform schlieren imagery on any flight vehicle which can navigate to the 
eclipse point. An airborne based system is currently under development to allow for schlieren imaging at close range for high altitude vehicles.

The technology described in this manuscript, Background Oriented Schlieren using Celestial Objects, is under United States Patent 9,599,497.

\section{Acknowledgements}

The authors thank the NASA Commercial Supersonics Technology (CST) Project for funding and support, NASA Armstrong Flight Research Center support personnel and researchers for enabling these flights, and U.S. Air Force Test Pilot School and NASA pilots for their skillful flying.

\section{References}

${ }^{1}$ Settles, G. S., Schlieren and Shadowgraph Techniques: Visualizing Phenomena in Transparent Media, Springer Science \& Business Media, New York, 2012.

${ }^{2}$ Dalziel, S. B., Hughes, G. O., and Sutherland, B. R., "Synthetic Schlieren," Proceedings of the 8th International Symposium on Flow Visualization, Sorrento, Italy, September 1-4, 1998.

${ }^{3}$ Raffel, M., Richard, H., and Meier G. E. A., "On the Applicability of Background Oriented Optical Tomography for Large Scale Aerodynamic Investigations," Experiments in Fluids, Vol. 28, No. 5, 2000, pp. 477-481.

${ }^{4}$ Raffel, M., Willert, C. E., and Kompenhans, J., Particle Image Velocimetry: A Practical Guide, Springer Science \& Business Media, New York, 2007.

${ }^{5}$ Atcheson, B., Heidrich, W., and Ihrke, I., "An Evaluation of Optical Flow Algorithms for Background Oriented Schlieren Imaging," Experiments in Fluids, Vol. 46, No. 3, 2009, pp. 467-476.

${ }^{6}$ Smith, N. T., Heineck, J. T., and Schairer, E. T., "Optical Flow for Flight and Wind Tunnel Background Oriented Schlieren Imaging," AIAA-2017-0472, 55th AIAA Aerospace Sciences Meeting, Grapevine, Texas, 2017.

${ }^{7}$ Hill, M. A., \& Haering, E. A., "Ground-to-Air Flow Visualization Using Solar Calcium-K Line Background-Oriented Schlieren," Experiments in Fluids, Vol. 58, No.1, 2017.

${ }^{8}$ Weinstein, L. M., "An Optical Technique for Examining Aircraft Shock Wave Structures in Flight," High-Speed Research: 1994 Sonic Boom Workshop: Atmospheric Propagation and Acceptability Studies, NASA CP-3279, 1994, pp. 1-17.

${ }^{9}$ Heineck, J. T., Banks, D. W., Schairer, E. T., Haering, E. A., and Bean, P. S., "Background Oriented Schlieren (BOS) of a Supersonic Aircraft in Flight," AIAA-2016-3356, AIAA Flight Testing Conference, Washington, D.C., 2016.

${ }^{10}$ Reda, I., "Solar Eclipse Monitoring for Solar Energy Applications," Solar Energy, Vol. 112, 2015, pp. 339-350.

${ }^{11}$ Vincenty, T. "Direct and Inverse Solutions of Geodesics on the Ellipsoid with Application of Nested Equations," Survey Review Vol. 23, No. 176, 2015, pp. 88-93

${ }^{12}$ Lucas, B. D., and Kanade, T., “An Iterative Image Registration Technique with an Application to Stereo Vision,” Proceedings of the $7^{\text {th }}$ International Joint Conference on Artificial Intelligence (IJCAI), Vancouver, British Columbia, Canada, 1981, pp. 674679 . 